\title{
OPTIMAL SYSTEM OF LIE ALGEBRA SUBALGEBRAS OF POINT SYMMETRIES GROUP FOR NONLINEAR HEAT EQUATION WITHOUT SOURCE
}

\author{
A.M. ILYASOV
}

\begin{abstract}
In this paper we construct an optimal system of subalgebras for the ninedimension Lie algebra of infinitesimal operators for a point symmetries group of a nonlinear heat equation with isotropic heat conductivity tensor and with a power dependence of the temperature. The results are presented as a lemma and a theorem. It is proven that up to transformations of internal automorphisms and some discrete automorphisms, there are 117 dissimilar subalgebras classes of various dimensions.
\end{abstract}

Keywords: nonlinear heat equation, Lie algebra, optimal system of subalgebras.

Mathematics Subject Classification: 35K59.

\section{INTRODUCTION}

By an appropriate change of variable, a closed system of equations describing isotropic spatial filtration of one-phase liquid in highly consolidated porous stratum [1] with the parameters of fluid and rock skeleton depending on threshold pressure can be reduced to a nonlinear threedimensional hear equation with the coefficients having power dependence on the temperature and the source depending on the temperature gradient. If one neglects gravitation forces in the filtration equations, then it reduced to the nonlinear heat equation without source

$$
\frac{\partial u}{\partial t}=\frac{\partial}{\partial x_{1}}\left(u^{\sigma} \frac{\partial u}{\partial x_{1}}\right)+\frac{\partial}{\partial x_{2}}\left(u^{\sigma} \frac{\partial u}{\partial x_{2}}\right)+\frac{\partial}{\partial x_{3}}\left(u^{\sigma} \frac{\partial u}{\partial x_{3}}\right) .
$$

In work [2], there was made a group classification of a spatial nonlinear heat equation with a source depending only on the temperature in the case of anisotropic, transversal-isotropic, and isotropic conductivity.

As it was shown in [2], in our case of isotropic heat conductivity corresponding to the widespread model of isotropic porous media, the kernel of main groups comprises shifts along the axes of four-dimensional space-time and the rotations around the axes. This kernel is associated with seven-dimensional Lie algebra of infinitesimal operators. It was shown in the same work that in the case of absence of the source and power dependence of the coefficients on the temperature, the Lie algebra $L_{7}$ enlarges to nine-dimensional algebra $L_{9}$ with a basis $(\sigma \neq 0)$

$$
\begin{gathered}
X_{1}=\frac{\partial}{\partial x_{1}}, \quad X_{2}=\frac{\partial}{\partial x_{2}}, \quad X_{3}=\frac{\partial}{\partial x_{3}}, \quad X_{4}=x_{3} \frac{\partial}{\partial x_{2}}-x_{2} \frac{\partial}{\partial x_{3}}, \\
X_{5}=x_{1} \frac{\partial}{\partial x_{3}}-x_{3} \frac{\partial}{\partial x_{1}}, \quad X_{6}=x_{2} \frac{\partial}{\partial x_{1}}-x_{1} \frac{\partial}{\partial x_{2}}, \quad X_{7}=\frac{\partial}{\partial t}, \\
X_{8}=2 t \frac{\partial}{\partial t}+x_{1} \frac{\partial}{\partial x_{1}}+x_{2} \frac{\partial}{\partial x_{2}}+x_{3} \frac{\partial}{\partial x_{3}},
\end{gathered}
$$

A.M. Ilyasov, Optimal System of Lie ALGEBRA SubAlgebras of the POINT Symmetries GRoup FOR NONLINEAR HEAT EQUATION WITHOUT SOURCE.

(C) Ilyasov A.M. 2013.

Submitted January 9, 2013. 


$$
X_{9}=\sigma x_{1} \frac{\partial}{\partial x_{1}}+\sigma x_{2} \frac{\partial}{\partial x_{2}}+\sigma x_{3} \frac{\partial}{\partial x_{3}}+2 u \frac{\partial}{\partial u} .
$$

Thus, the shift and rotation operators are supplemented by the operator of simultaneous dilatation in the space-time as well as by the operator of simultaneous dilatation in spatial variables and dependent variable.

In what follows, we shall construct an optimal system of Lie algebra $L_{9}$ subalgebbras (OSS) which chosen basis (2). Linear transformations for constructing the optimal system are considered over the field of real numbers, since we are interested in real group solutions to equation (11).

\section{Group of internal automorphisms for Lie algebra $L_{9}$}

First let us calculate the commutators of basis operators for algebra $L_{9}$. By definition, the commutator of basis operators (2) $X_{i}$ and $X_{j}, i, j=1, \ldots, 9$, is the operator [3]

$$
\left[X_{i}, X_{j}\right]=X_{i} X_{j}-X_{j} X_{i}, \quad i, j=1, \ldots, 9 .
$$

The calculations by formula (3) are presented in Table 1 of commutators for operators of algebra $L_{9}$. For the sake of brevity, the operators are replaced by numbers in the table. For instance, $-(\sigma) 1$ indicates $-\sigma X_{1}$. In the first columns and rows in Table 1 we provide basis operators, while on their intersections we give their commutators.

Table 1

\begin{tabular}{|l|l|l|l|l|l|l|l|l|l|}
\hline & $\mathbf{1}$ & $\mathbf{2}$ & $\mathbf{3}$ & $\mathbf{4}$ & $\mathbf{5}$ & $\mathbf{6}$ & $\mathbf{7}$ & $\mathbf{8}$ & $\mathbf{9}$ \\
\hline $\mathbf{1}$ & 0 & 0 & 0 & 0 & 3 & -2 & 0 & 1 & $(\sigma) 1$ \\
\hline $\mathbf{2}$ & 0 & 0 & 0 & -3 & 0 & 1 & 0 & 2 & $(\sigma) 2$ \\
\hline $\mathbf{3}$ & 0 & 0 & 0 & 2 & -1 & 0 & 0 & 3 & $(\sigma) 3$ \\
\hline $\mathbf{4}$ & 0 & 3 & -2 & 0 & 6 & -5 & 0 & 0 & 0 \\
\hline $\mathbf{5}$ & -3 & 0 & 1 & -6 & 0 & 4 & 0 & 0 & 0 \\
\hline $\mathbf{6}$ & 2 & -1 & 0 & 5 & -4 & 0 & 0 & 0 & 0 \\
\hline $\mathbf{7}$ & 0 & 0 & 0 & 0 & 0 & 0 & 0 & $(2) 7$ & 0 \\
\hline $\mathbf{8}$ & -1 & -2 & -3 & 0 & 0 & 0 & $-(2)$ & 0 & 0 \\
\hline $\mathbf{9}$ & $-(\sigma) 1$ & $-(\sigma) 2$ & $-(\sigma) 3$ & 0 & 0 & 0 & 0 & 0 & 0 \\
\hline
\end{tabular}

We write an arbitrary element of algebra $L_{9}$ in terms of basis (2) as $X=x^{i} X_{i},(i=1, \ldots, 9)$. We denote by $P_{1}(x)=\left(x^{1}, x^{2}, x^{3}\right)$ and $P_{2}(x)=\left(x^{4}, x^{5}, x^{6}\right)$ the projections of the coordinate vector $\vec{x}$ for the element $X$. Then, in accordance with introduced notations, the coordinate vector of an arbitrary one-dimensional operator in algebra $L_{9}$ can be represented as

$$
\vec{x}=\left(P_{1}(x), P_{2}(x), x^{7}, x^{8}, x^{9}\right) .
$$

Let $X^{\prime}=x^{i} X_{i},(i=1, \ldots, 9)$ be an operator in algebra $L_{9}$ in basis (2) and $Y \in L_{9}$ be another infinitesimal operator written in terms of the same basis. Let us calculate the group of internal automorphisms of algebra $L_{9}$. For any operator $Y \in L_{9}$, the one-parametric group of internal automorphisms solves problem [3],

$$
\frac{\partial X^{\prime}}{\partial a}=\left[X^{\prime}, Y\right], \quad X^{\prime}(0)=X
$$

As it was shown in [3], in order to calculate all internal automorphisms, it is sufficient to calculate one-parametric groups of internal automorphisms for basis operators. Hence, the internal automorphisms are found by the solutions to the equation

$$
\frac{\partial x^{i}}{\partial a_{j}} X_{i}=x^{i}\left[X_{i}, X_{j}\right], \quad x^{i}(0)=x^{i}, \quad i, j=1, \ldots, 9,
$$

where in (5), instead of operator $Y$, one should take subsequently all the basis operators $X_{j}$. 
Let $Y=X_{1}$, then by (6) and the commutators table we have

$$
\frac{\partial x^{\prime i}}{\partial a_{1}} X_{i}=x^{\prime i}\left[X_{i}, X_{1}\right]=-x^{\prime 5} X_{3}+x^{6} X_{2}-x^{\prime 8} X_{1}-x^{\prime 9} \sigma X_{1}, \quad x^{\prime i}(0)=x^{i}, \quad i=1, \ldots, 9,
$$

or the following Cauchy problem for ODEs system determining coordinates $x^{i}$,

$$
\left\{\begin{array}{l}
\frac{\partial x^{\prime 1}}{\partial a_{1}}=-x^{\prime 8}-\sigma x^{\prime 9}, \quad \frac{\partial x^{\prime 2}}{\partial a_{1}}=x^{\prime 6}, \quad \frac{\partial x^{\prime 3}}{\partial a_{1}}=-x^{\prime 5}, \quad \frac{\partial x^{\prime i}}{\partial a_{1}}=0 \\
x^{\prime 1}(0)=x^{1}, \quad x^{\prime 2}(0)=x^{2}, \quad x^{\prime 3}(0)=x^{3}, \quad x^{\prime i}(0)=x^{i}, \quad i=4, \ldots, 9 .
\end{array}\right.
$$

It yields the following solution to this problem,

$$
\left\{\begin{array}{l}
x^{\prime 1}=-\left(x^{8}+\sigma x^{9}\right) a_{1}+x^{1} \\
x^{\prime 2}=x^{6} a_{1}+x^{2} \\
x^{\prime 3}=-x^{5} a_{1}+x^{3} \\
x^{\prime i}=x^{i}, \quad i=4, \ldots, 9
\end{array}\right.
$$

As in (17), by (6) and the commutators table, we obtain one-parametric groups for operators $Y=X_{2}$ and $Y=X_{3}$. It is easy to show that the composition of obtained one-parametric automorphisms groups determines a three-parametric transformation of the projection $P_{1}(x)$,

$$
\Gamma: P_{1}\left(x^{\prime}\right)=P_{1}(x)+P_{2}(x) \times \vec{\alpha}-\left(x^{8}+\sigma x^{9}\right) \vec{\alpha},
$$

where $x^{\prime}$ stands for the transformed coordinates, $\vec{\alpha}=\left(a_{4}, a_{5}, a_{6}\right)$ is the parametric vector, and ii $\times$ i $i$ denotes the vector product.

By (6) we find one-parametric group of internal automorphisms of Lie algebra $L_{9}$ for the operator $Y=X_{4}$, which is simultaneous rotations around axes $O x^{1}$ and $O x^{4}$,

$$
\left\{\begin{array}{l}
x^{\prime 2}=\sin a_{4} \cdot x^{3}+\cos a_{4} \cdot x^{2} \\
x^{\prime 3}=\cos a_{4} \cdot x^{3}-\sin a_{4} \cdot x^{2} \\
x^{\prime 5}=\sin a_{4} \cdot x^{6}+\cos a_{4} \cdot x^{5} \\
x^{\prime 6}=\cos a_{4} \cdot x^{6}-\sin a_{4} \cdot x^{5} \\
x^{i}=x^{i}, \quad i=1,4,7,8,9
\end{array}\right.
$$

In the same way as in (8), as $Y=X_{5}$, by (6) we get the group of simultaneous rotations around the axes $O x^{2}$ and $O x^{5}$, while as $Y=X_{6}$ we obtain the group of simultaneous rotations around the axes $O x^{3}$ and $O x^{6}$. The composition of one-parametric rotation groups determine three-parametric transformation of rotations $O$ for the projections $P_{1}(x)$ and $P_{2}(x)$ by the formulae,

$$
P_{1}\left(x^{\prime}\right)=O P_{1}(x), \quad P_{2}\left(x^{\prime}\right)=O P_{2}(x), \quad O O^{T}=I, \quad \operatorname{det} O=1 .
$$

As $Y=X_{7}$, by (6) we obtain the transformation which will be indicated by

$$
\Pi: \quad x^{\prime 7}=-2 a_{7} x^{8}+x^{7} .
$$

As $Y=X_{8}$, we get the one-parametric group of simultaneous dilatation of projection $P_{1}(x)$ and coordinate $x^{7}$. This transformation will be indicated by

$$
R_{1}: \quad P_{1}\left(x^{\prime}\right)=a_{8} P_{1}(x), \quad x^{\prime 7}=a_{8}^{2} x^{7} .
$$

As $Y=X_{9}$, by (6) we calculate the one-parametric group of homogeneous dilatations of projections $P_{1}(x)$ which will be denoted by

$$
R_{2}: \quad P_{1}\left(x^{\prime}\right)=a_{9} P_{1}(x) .
$$

The composition of obtained transformation group forms a nine-parametric internal automorphisms group.

Let us consider how the internal automorphisms act on various coordinate projection of vector $\vec{x}$ (4). The following automorphisms groups act on the projections of coordinate vector $\vec{x}$ : only rotations $O$ act on projections $P_{2} O$; rotations $O$, transformation $\Gamma$, and dilatations $R_{1}$ and $R_{2}$ 
act on projection $P_{1}$; coordinate $x^{7}$ changes only under the transformation $\Pi$ and dilatation $R_{1}$; and no internal automorphisms act on the projection defined by coordinates $x_{8}, x_{9}$.

Moreover, by considering Table 1, we observe the following discrete automorphisms,

$$
\begin{gathered}
\varepsilon_{1}: \quad P_{1}\left(x^{\prime}\right)=-P_{1}(x), \\
\varepsilon_{2}: \quad x^{\prime 7}=-x^{7} .
\end{gathered}
$$

\section{Optimal Systems of SUbAlgebras}

In work [4, there was described an algorithm of calculating an optimal system for an arbitrary Lie algebra subalgebras and there were given examples of calculating some subalgebras of finite-dimensional algebras for a linear one-dimensional heat equation, gas dynamics equations and motion equations for an isotropic non-compressible liquid with the viscosity and heat conductivity coefficients depending on the temperature. For the models of gas dynamics, the survey of constructed optimal system of subalgebras was provided in work [5]. Let us apply this algorithm for calculating the optimal subalgebras system for algebra $L_{9}$ of spatial nonlinear heat equations without the source and with an isotropic heat tensor having power dependence on the temperature.

Consider Table 1 of commutators for basis operators of algebra $L_{9}$. We see that the fourdimensional space spanned over the basis vectors $\left\{X_{1}, X_{2}, X_{3}, X_{9}\right\}$, is ideal $J_{4}$ of algebra $L_{9}$, while the five-dimensional space spanned over vectors $\left\{X_{4}, X_{5}, X_{6}, X_{7}, X_{8}\right\}$, is subalgebra $L_{5}$ of algebra $L_{9}$. In its turn, subalgebra $L_{5}$ can be expanded into the direct sum of its ideals $J_{51}=\left\{X_{4}, X_{5}, X_{6}\right\}$ and $J_{52}=\left\{X_{7}, X_{8}\right\}$. Thus, the expansion

$$
L_{9}=L_{5} \dot{\oplus} J_{4}=\left(J_{51} \oplus J_{52}\right) \dot{\oplus} J_{4}
$$

holds true.

We denote the coordinates of one-dimensional subalgebras by $x^{i}$, the notations $x^{i}, y^{i}$ stand for two-dimensional subalgebras, $x^{i}, y^{i}, z^{i}$ are used for three-dimensional subalgebras, $x^{i}, y^{i}, z^{i}, w^{i}$ are employed for four-dimensional subalgebras and so forth.

\section{Optimal SYSTEM OF SUbALGEBRAS FOR ALGEBRA $L_{5}$}

Let us calculate the optimal system of subalgebras for algebra $L_{5}$. In accordance with expansion (9), algebra is the direct sum of its ideals $J_{51}$ and $J_{52}$,

$$
L_{5}=\left\{X_{4}, X_{5}, X_{6}\right\} \oplus\left\{X_{7}, X_{8}\right\}=J_{51} \oplus J_{52}
$$

Employing the algorithm described in work [4], we calculate first OSS for ideal $J_{51}$, and then, according to (10), to each operator in OSS of ideal $J_{51}$ (including zero operator) we shall add a linear combination of basis operators of ideal $J_{52}$ completing operators from OSS for ideal $J_{51}$ to operators in algebra $L_{5}$. Then, by means of internal automorphisms, we shall reduce the operators in subalgebras in $L_{5}$ to the simplest form (making minimal amount of arbitrary coordinates for operators). In this way we find OSS for algebra $L_{5}$, each its representative has as a projection one of the subalgebras in OSS for ideal $J_{51}$ or zero projection.

First we find OSS for ideal $J_{51}$. One-dimensional subalgebra is an arbitrary operator in ideal $J_{51}$ whose basis operator reads as $X=x^{4} X_{4}+x^{5} X_{5}+x^{6} X_{6}$. This operator can be represented as a matrix-column for coordinates of basis operator $\left(\begin{array}{lll}x^{4} & x^{5} & x^{6}\end{array}\right)$. Applying rotation $O$ and changing the basis, we obtain $\left(\begin{array}{cll}\varepsilon & 0 & 0\end{array}\right)$. If $\varepsilon=0$, we obtain the zero subalgebra, and if $\varepsilon=1$, we get the one-dimensional subalgebra $\left\{X_{4}\right\}$.

Consider now an arbitrary two-dimensional subalgebra for ideal $J_{51}$ which can be represented as the matrix for the coordinates of basis operators

$$
\left(\begin{array}{lll}
x^{4} & x^{5} & x^{6} \\
y^{4} & y^{5} & y^{6}
\end{array}\right)
$$


Applying transformation $O$ and changing the basis, we reduce the matrix to the form

$$
\left(\begin{array}{lll}
1 & 0 & 0 \\
0 & 1 & 0
\end{array}\right)
$$

However, for such basis the condition of subalgebra $\left[X_{4}, X_{5}\right]=X_{6} \neq \lambda X_{4}+\mu X_{5}$ does not hold. Thus, there exist no two-dimensional subalgebras in OSS for ideal $J_{51}$.

A basis of three-dimensional subalgebra in $J_{51}$ can be represented as a real non-degenerate matrix

$$
\left(\begin{array}{lll}
x^{4} & x^{5} & x^{6} \\
y^{4} & y^{5} & y^{6} \\
z^{4} & z^{5} & z^{6}
\end{array}\right)
$$

Then by changing the basis, we can reduce this matrix to the unit one. We obtain the threedimensional subalgebra, $\left\{X_{4}, X_{5}, X_{6}\right\}$. The subalgebra condition holds true. Hence, OSS for ideal $J_{51}$ consists of three subalgebras being one-dimensional, three-dimensional, and zero.

Let us calculate all subalgebra in $L_{5}$ having zero projection on $J_{51}$. One-dimensional subal-

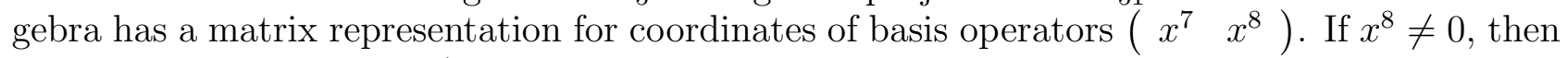
transformation $\Pi$ makes $x^{7}=0$, and the change of the basis leads us to the subalgebra $\left\{X_{8}\right\}$. If $x^{8}=0$, then by the change of the basis we arrive at the subalgebra $\left\{X_{7}\right\}$. Thus, there exist two one-dimensional subalgebras in $L_{5}$ with zero projection on $J_{51}$.

Two-dimensional subalgebra with zero projection reads as $\left\{X_{7}, X_{8}\right\}$. There is no subalgebras of dimension higher than two with zero projection, since all the operators become linearly dependent. Thus, in $L_{5}$ there are zero, two one-dimensional and one two-dimensional subalgebras with zero projection on $J_{51}$.

One-dimensional subalgebra in $L_{5}$ with the projection $\left\{X_{4}\right\}$ on $J_{51}$ has the matrix representation of operators coordinates $\left(\begin{array}{lll}1 & x^{7} & x^{8}\end{array}\right)$. If $x^{8} \neq 0$, then transformation $\Pi$ makes $x^{7}=0$, and the change of the basis leads us to the subalgebra $\left\{X_{4}+\alpha X_{8}\right\}$. If $x^{8}=0$, then the transformations $R_{1}$ and $\varepsilon_{2}$ give the subalgebra $\left\{X_{4}+X_{7}\right\}$. Thus, there are two one-dimensional subalgebras in $L_{5}$ with the projection $\left\{X_{4}\right\}$ on $J_{51}$.

Two-dimensional subalgebra in $L_{5}$ with the projection $\left\{X_{4}\right\}$ on $J_{51}$ has the matrix representation of operators coordinates

$$
\left(\begin{array}{lll}
1 & x^{7} & x^{8} \\
0 & y^{7} & y^{8}
\end{array}\right)
$$

If $y^{8} \neq 0$, then transformation $\Pi$ makes $y^{7}=0$, the change of the basis gives $y^{8}=1, x^{8}=0$, and transformations $R_{1}$ and $\varepsilon_{2}$ lead us to subalgebra $\left\{X_{4}+\varepsilon X_{7}, X_{8} ; \varepsilon=0,1\right\}$. In the case $\varepsilon=1$, subalgebra condition does not hold, since $\left[X_{4}+X_{7}, X_{8}\right]=2 X_{7} \neq \lambda\left(X_{4}+X_{7}\right)+\mu X_{8}$. If $\varepsilon=0$, we obtain subalgebra $\left\{X_{4}, X_{8}\right\}$. If $y^{8}=0$, then the change of the basis imply $y^{7}=1, x^{7}=0$ that gives subalgebra $\left\{X_{4}+\alpha X_{8}, X_{7}\right\}$. Thus, there exist two two-dimensional subalgebras in $L_{5}$ with the projection $\left\{X_{4}\right\}$ on $J_{51}$.

There is just one three-dimensional subalgebra in $L_{5}$ with the projection $\left\{X_{4}\right\}$ on $J_{51}$, $\left\{X_{4}, X_{7}, X_{8}\right\}$.

Three-dimensional subalgebra in $L_{5}$ with the projection $\left\{X_{4}, X_{5}, X_{6}\right\}$ in $J_{51}$ has the matrix representation of operators coordinates

$$
\left(\begin{array}{lllll}
1 & 0 & 0 & x^{7} & x^{8} \\
0 & 1 & 0 & y^{7} & y^{8} \\
0 & 0 & 1 & z^{7} & z^{8}
\end{array}\right) .
$$

Calculating all possible commutators of subalgebra, we find that subalgebra condition holds true if and only if $x^{7}=y^{7}=z^{7}=x^{8}==y^{8}=z^{8}=0$. That is, we obtain the original projection $\left\{X_{4}, X_{5}, X_{6}\right\}$. 
Four-dimensional subalgebra in $L_{5}$ with the projection $\left\{X_{4}, X_{5}, X_{6}\right\}$ on $J_{51}$ has the matrix representation of operators coordinates

$$
\left(\begin{array}{ccccc}
1 & 0 & 0 & x^{7} & x^{8} \\
0 & 1 & 0 & y^{7} & y^{8} \\
0 & 0 & 1 & z^{7} & z^{8} \\
0 & 0 & 0 & w^{7} & w^{8}
\end{array}\right) .
$$

If $w^{8} \neq 0$, then transformation $\Pi$ makes $w^{7}=0$, the change of the basis gives $w^{8}=1, x^{8}=$ $y^{8}=z^{8}=0$, and subalgebra conditions imply $x^{7}=y^{7}=z^{7}=0$ and lead us to the subalgebra $\left\{X_{4}, X_{5}, X_{6}, X_{8}\right\}$. If $w^{8}=0$, by change of the basis we obtain $w^{7}=1, x^{7}=y^{7}=z^{7}=0$, while subalgebra condition yield $x^{8}=y^{8}=z^{8}=0$. We arrive at the subalgebra $\left\{X_{4}, X_{5}, X_{6}, X_{7}\right\}$.

A five-dimensional subalgebra in $L_{5}$ with the projection $\left\{X_{4}, X_{5}, X_{6}\right\}$ on $J_{51}$ is $L_{5}$. Thus, there exist one three-dimensional, two four-dimensional and one five-dimensional subalgebra with the projection $\left\{X_{4}, X_{5}, X_{6}\right\}$ on $J_{51}$.

Hence, we have proven the following lemma.

Lemma 1. Optimal system of subalgebras for Lie algebra $L_{5}$ with commutators in Table 1 comprises 12 classes of dissimilar subalgebras up to internal automorphisms and discrete automorphisms $\varepsilon_{1}$ and $\varepsilon_{2}$. The classes are presented in Table 2.

Table 2.

\begin{tabular}{|l|l|l||l|l|l|}
\hline $\mathrm{r}$ & $\mathrm{i}$ & Basis & $\mathrm{r}$ & $\mathrm{i}$ & \multicolumn{2}{|l|}{ Basis } \\
\hline \hline 1 & 1 & $4+\alpha 8$ & 3 & 1 & $4,5,6$ \\
\hline & 2 & $4+7$ & & 2 & $4,7,8$ \\
\hline & 3 & 7 & 4 & 1 & $4,5,6,7$ \\
\hline & 4 & 8 & & 2 & $4,5,6,8$ \\
\hline \hline 2 & 1 & 4,8 & 5 & 1 & $\begin{array}{l}4,5,6,7, \\
8\end{array}$ \\
\hline & 2 & $4+\alpha 8 ; 7$ & & & \\
\hline & 3 & 7,8 & & & \\
\hline
\end{tabular}

In the table $r$ is the dimension of a subalgebra, $i$ is the sequence number of a subalgebra in a given dimension, $\alpha$ is an arbitrary constant.

\section{OptimAL SYSTEM OF SUBALGEBRAS FOR ALGEBRA $L_{9}$}

According to (9), algebra $L_{9}$ is the semi-direct sum of subalgebra $L_{5}$ and ideal $J_{4}$,

$$
L_{9}=L_{5} \dot{\oplus} J_{4}=\left\{X_{4}, X_{5}, X_{6}, X_{7}, X_{8}\right\} \dot{\oplus}\left\{X_{1}, X_{2}, X_{3}, X_{9}\right\} .
$$

Proceeding as above, by the proven lemma one can construct OSS for algebra $L_{9}$ taking subalgebras in Table 2 as the projections on $L_{5}$ and adding to their operators the parts of operators in ideal $J_{4}$.

We first determine OSS for ideal $J_{4}$ which coincides with subalgebras in the optimal system of algebra $L_{9}$ having zero projection on $L_{5}$. One-dimensional subalgebras in $L_{9}$ with zero projection on $L_{5}$ possesses the matrix representation $\left(\begin{array}{llll}x^{1} & x^{2} & x^{3} & x^{9}\end{array}\right)$. If $x^{9} \neq 0$, transformation $\Gamma$ makes $P_{1}(x)=0$, and change of the basis gives subalgebra $\left\{X_{9}\right\}$. If $x^{9}=0$, then the rotation $O$ and change of the basis lead us to subalgebra $\left\{X_{1}\right\}$. Thus, there are two one-dimensional subalgebras in $L_{9}$ with zero projection on $L_{5}:\left\{X_{1}\right\}$ is subalgebra 1.1 in Table 3 and $\left\{X_{9}\right\}$ is subalgebra 1.2 in Table 3.

Two-dimensional subalgebras in $L_{9}$ with zero projection on $L_{5}$ have matrix representation

$$
\left(\begin{array}{llll}
x^{1} & x^{2} & x^{3} & x^{9} \\
y^{1} & y^{2} & y^{3} & y^{9}
\end{array}\right) .
$$


If $y^{9} \neq 0$, then transformation $\Gamma$ makes $P_{1}(y)=0$, and change of the basis gives $y^{9}=1, x^{9}=0$. Rotation $O$ makes $P_{1}(x)=\left(\begin{array}{lll}x^{1} & 0 & 0\end{array}\right)$. After change of the basis we obtain subalgebra $\left\{X_{1}, X_{9}\right\}$. If $y^{9}=0$, then rotation $O$ makes $P_{1}(y)=\left(\begin{array}{lll}y^{1} & 0 & 0\end{array}\right)$, change of the basis gives $y^{1}=1, x^{1}=0$. Rotation $O$ around axis $O x^{1}$ implies $x^{3}=0$. If $x^{9} \neq 0$, transformation $\Gamma$ makes $P_{1}(x)=0$, while change of the basis gives $x^{9}=1$, and we arrive at the subalgebra obtained above. If $x^{9}=0$, then change of the basis makes $x^{2}=1$ and we obtain subalgebra $\left\{X_{1}, X_{2}\right\}$. Hence, there are two two-dimensional subalgebras in $L_{9}$ with zero projection on $L_{5}:\left\{X_{1}, X_{9}\right\}$ is subalgebra 2.2 in Table 3 and $\left\{X_{1}, X_{2}\right\}$ is subalgebra 2.1 in Table 3.

Three-dimensional subalgebras in $L_{9}$ with zero projection on $L_{5}$ have matrix representation

$$
\left(\begin{array}{llll}
x^{1} & x^{2} & x^{3} & x^{9} \\
y^{1} & y^{2} & y^{3} & y^{9} \\
z^{1} & z^{2} & z^{3} & z^{9}
\end{array}\right) .
$$

If $z^{9} \neq 0$, transformation $\Gamma$ makes $P_{1}(z)=0$, and change of the basis gives $z^{9}=1, x^{9}=y^{9}=0$. Rotation $O$ makes $P_{1}(y)=\left(\begin{array}{lll}y^{1} & 0 & 0\end{array}\right)$. Rotation $O$ around axis $O x^{1}$ gives $x^{3}=0$, while change of the basis makes $y^{1}=1$. We obtain subalgebra $\left\{X_{1}, X_{2}, X_{9}\right\}$. If $z^{9}=0$, then rotation $O$ yields $P_{1}(z)=\left(\begin{array}{ccc}z^{1} & 0 & 0\end{array}\right)$, and change of the basis implies $z^{1}=1, x^{1}=y^{1}=0$. Rotation $O$ around the axis $O x^{1}$ makes $x^{3}=0$, and subalgebra matrix casts into the form

$$
\left(\begin{array}{cccc}
0 & x^{2} & 0 & x^{9} \\
0 & y^{2} & y^{3} & y^{9} \\
1 & 0 & 0 & 0
\end{array}\right) .
$$

If $x^{9} \neq 0$, transformation $\Gamma$ makes $x^{2}=0$, and change of the basis gives $x^{9}=1, y^{9}=0$. Rotation $O$ around axis $O x^{1}$ implies $y^{3}=0$, and change of the basis yields $y^{2}=1$. We arrive at the subalgebra obtained above. If $x^{9}=0$, then change of the basis makes $x^{2}=1, y^{2}=0$. If $y^{9} \neq 0$, then transformation $\Gamma$ gives $y^{3}=0$ and we again obtain the same subalgebra. If $y^{9}=0$, change of the basis makes $y^{3}=1$ and we obtain subalgebra $\left\{X_{1}, X_{2}, X_{3}\right\}$. Hence, there exist two three-dimensional subalgebras in $L_{9}$ with zero projection on $L_{5}:\left\{X_{1}, X_{2}, X_{9}\right\}$ is subalgebra 3.2 in Table 3 and $\left\{X_{1}, X_{2}, X_{3}\right\}$ is subalgebra 3.1 in Table 3.

Four-dimensional subalgebra in $L_{9}$ with zero projection on $L_{5}$ coincides with ideal $J_{4}$, it is subalgebra 4.1 in Table 3.

In order to calculate other subalgebras in OSS of algebra $L_{9}$, in accordance with (11), to each subalgebra in Lemma we should add the parts of the operators in ideal $J_{4}$. At that, the onedimensional projection on the space $L_{5}$ have subalgebras $\mathrm{H}$ of dimension $\operatorname{dim} \mathrm{H}=1 \div 5$. There exist no subalgebras of higher dimensions with one-dimensional projections on subspace $L_{5}$, since the operators become linearly dependent. In the same way, two-dimensional projections on space $L_{5}$ belong to subalgebras $\mathrm{H}$ of dimension $\operatorname{dim} \mathrm{H}=2 \div 6$; three-dimensional projections belong to subalgebras of dimension $\operatorname{dim} \mathrm{H}=3 \div 7$; four-dimensional projections belong to subalgebras of dimension $\operatorname{dim} \mathrm{H}=4 \div 8$; five-dimensional projections belong to subalgebras of dimension $\operatorname{dim} \mathrm{H}=5 \div 9$. In the work we shall provide the examples of calculating subalgebras of all dimensions in factful cases. The results of all calculations are provided in Table 3.

Consider one-dimensional subalgebra 1.1 in Table 2. One-dimensional subalgebras in $L_{9}$ with the projection $\left\{X_{4}+\alpha X_{8}\right\}$ on space $L_{5}$ have the following matrix representation $\left(\begin{array}{llllll}1 & \alpha & x^{1} & x^{2} & x^{3} & x^{9}\end{array}\right)$. If $\alpha+\sigma x^{9} \neq 0$, transformation $\Gamma$ makes $P_{1}(x)=0$ and we obtain the following subalgebra $\left\{X_{4}+\alpha X_{8}+\beta X_{9}, \beta \neq-\alpha / \sigma\right\}$. If $\alpha+\sigma x^{9}=0$, after the change of the parameter $\alpha / \sigma \rightarrow \alpha$ transformation $\Gamma$ gives $P_{1}(x)=\left(\begin{array}{lll}x^{1} & 0 & 0\end{array}\right)$ and we get subalgebra $\left\{X_{4}+\sigma \alpha X_{8}+\varepsilon X_{1}-\alpha X_{9}\right\}$. If $\varepsilon=0$, this subalgebra can be joined with the previous one. We obtain subalgebra with no restriction for the values of the parameter $\left\{X_{4}+\alpha X_{8}+\beta X_{9}\right\}$. If $\varepsilon=1$, we obtain the following subalgebra $\left\{X_{4}+X_{1}+\alpha\left(\sigma X_{8}-X_{9}\right)\right\}$. Thus, there exist two one-dimensional subalgebras in $L_{9}$ with the projection $\left\{X_{4}+\alpha X_{8}\right\}$ on $L_{5}:\left\{X_{4}+\alpha X_{8}+\beta X_{9}\right\}$ is subalgebra 1.3 in Table 3 and $\left\{X_{4}+X_{1}+\alpha\left(\sigma X_{8}-X_{9}\right)\right\}$ is subalgebra 1.4 in Table 3. 
Two-dimensional subalgebras in $L_{9}$ with the projection $\left\{X_{4}+\alpha X_{8}\right\}$ on subspace $L_{5}$ have matrix representation

$$
\left(\begin{array}{llllll}
1 & \alpha & x^{1} & x^{2} & x^{3} & x^{9} \\
0 & 0 & y^{1} & y^{2} & y^{3} & y^{9}
\end{array}\right)
$$

If $y^{9} \neq 0$, transformation $\Gamma$ makes $P_{1}(y)=0$, change of the basis gives $y^{9}=1, x^{9}=0$. Rotation $O$ around axis $O x^{1}$ implies $x^{3}=0$, and by subalgebra conditions $\left[X_{4}+\alpha X_{8}+x^{1} X_{1}+\right.$ $\left.x^{2} X_{2}, X_{9}\right]=\sigma x^{1} X_{1}+\sigma x^{2} X_{2}=0$ it follows that it is possible as $x^{1}=x^{2}=0$. We obtain subalgebra $\left\{X_{4}+\alpha X_{8}, X_{9}\right\}$. If $y^{9}=0$, transformation $\Gamma$ makes $P_{1}(x)=\left(\begin{array}{lll}x^{1} & 0 & 0\end{array}\right)$, and rotation $O$ around axis $O x^{1}$ gives $y^{3}=0$. Subalgebra condition imply $y^{2}=0$, change of the basis gives $y^{1}=1, x^{1}=0$. We get subalgebra $\left\{X_{4}+\alpha X_{8}+\beta X_{9}, X_{1}\right\}$. Thus, there exist two two-dimensional subalgebras in $L_{9}$ with the projection $\left\{X_{4}+\alpha X_{8}\right\}$ on $L_{5}:\left\{X_{4}+\alpha X_{8}, X_{9}\right\}$ is subalgebra 2.3 in Table 3 and $\left\{X_{4}+\alpha X_{8}+\beta X_{9}, X_{1}\right\}$ is subalgebra 2.4 in Table 3.

Three-dimensional subalgebras in $L_{9}$ with the projection $\left\{X_{4}+\alpha X_{8}\right\}$ on subspace $L_{5}$ have matrix representation

$$
\left(\begin{array}{cccccc}
1 & \alpha & x^{1} & x^{2} & x^{3} & x^{9} \\
0 & 0 & y^{1} & y^{2} & y^{3} & y^{9} \\
0 & 0 & z^{1} & z^{2} & z^{3} & z^{9}
\end{array}\right)
$$

If $z^{9} \neq 0$, transformation $\Gamma$ makes $P_{1}(z)=0$, change of the basis gives $z^{9}=1, x^{9}=y^{9}=0$. Rotation $O$ around axis $O x^{1}$ makes $x^{3}=0$. Subalgebra condition for commutator of operators 1 and 2 imply the homogeneous system of equations

$$
\left\{\begin{array}{l}
y^{2}(\lambda+\alpha)+y^{3}=0 \\
y^{2}-(\lambda+\alpha) y^{3}=0
\end{array}\right.
$$

with the determinant $\Delta=-(\lambda+\alpha)^{2}-1 \neq 0$ that implies $y^{2}=y^{3}=0$. Change of the basis makes $y^{1}=1, x^{1}=0$. We obtain subalgebra $\left\{X_{4}+\alpha X_{8}, X_{1}, X_{9}\right\}$. If $y^{9} \neq 0$, replacing two last columns, we arrive to the considered above case. If $y^{9}=z^{9}=0$, transformation $\Gamma$ makes $P_{1}(x)=\left(\begin{array}{lll}x^{1} & 0 & 0\end{array}\right)$, and rotation $O$ around axis $O x^{1}$ gives $y^{3}=0$. We obtain the following representation for subalgebra

$$
\left(\begin{array}{cccccc}
1 & \alpha & x^{1} & 0 & 0 & x^{9} \\
0 & 0 & y^{1} & y^{2} & 0 & 0 \\
0 & 0 & z^{1} & z^{2} & z^{3} & 0
\end{array}\right)
$$

Subalgebra condition for the commutator of operators 1 and 2 yields the system of equations

$$
\left\{\begin{array}{l}
\mu z^{1}=-\left(\lambda+\alpha+\sigma x^{9}\right) y^{1} \\
\mu z^{2}=-\left(\lambda+\alpha+\sigma x^{9}\right) y^{2} \\
\mu z^{3}=y^{2}
\end{array}\right.
$$

There exist two options.

1) $y^{2} \neq 0$. The latter equation in the system implies $\mu \neq 0, z^{3} \neq 0$. Then change of the basis makes $y^{2}=1, z^{2}=0, z^{3}=1$ and the first equation yields $z^{1}=0$. Subalgebra conditions for the commutators of the first and third operators follows $y^{1}=0$. We obtain subalgebra $\left\{X_{4}+\alpha X_{8}+\varepsilon X_{1}+\beta X_{9}, X_{2}, X_{3}\right\}$. If $\varepsilon=0$, we have subalgebra $\left\{X_{4}+\alpha X_{8}+\beta X_{9}, X_{2}, X_{3}\right\}$. If $\varepsilon=1$ and $\alpha+\sigma \beta \neq 0$, then transformation $\Gamma$ makes $x^{1}=0$. We obtain subalgebra $\left\{X_{4}+\alpha X_{8}+\beta X_{9}, X_{2}, X_{3} ; \beta \neq-\alpha / \sigma\right\}$ which is embedded into the previous one. If $\varepsilon=1$ and $\alpha+\sigma \beta=0$, we have subalgebra $\left\{X_{4}+X_{1}+\alpha\left(\sigma X_{8}-X_{9}\right), X_{2}, X_{3}\right\}$.

2) $y^{2}=0$. Change of the basis makes $y^{1}=1, x^{1}=z^{1}=0$, and subalgebra conditions for the first and third operators implie $z^{2}=z^{3}=0$. That is, in this case the operators are linearly dependent. Thus, there exist three three-dimensional subalgebras in $L_{9}$ with the projection $\left\{X_{4}+\alpha X_{8}\right\}$ on $L_{5}:\left\{X_{4}+\alpha X_{8}, X_{1}, X_{9}\right\}$ is subalgebra 3.3 in Table $3,\left\{X_{4}+\alpha X_{8}+\beta X_{9}, X_{2}, X_{3}\right\}$ is subalgebra 3.4 in Table 3 and $\left\{X_{4}+X_{1}+\alpha\left(\sigma X_{8}-X_{9}\right), X_{2}, X_{3}\right\}$ is subalgebra 3.5 in Table 3. 
Four-dimensional subalgebras in $L_{9}$ with the projection $\left\{X_{4}+\alpha X_{8}\right\}$ on subspace $L_{5}$ have matrix representation

$$
\left(\begin{array}{cccccc}
1 & \alpha & x^{1} & x^{2} & x^{3} & x^{9} \\
0 & 0 & y^{1} & y^{2} & y^{3} & y^{9} \\
0 & 0 & z^{1} & z^{2} & z^{3} & z^{9} \\
0 & 0 & w^{1} & w^{2} & w^{3} & w^{9}
\end{array}\right)
$$

There possible two cases.

1) If $w^{9} \neq 0$, transformation $\Gamma$ makes $P_{1}(w)=0$, change of the basis gives $w^{9}=1, x^{9}=$ $y^{9}=z^{9}=0$. Rotation $O$ around axis $O x^{1}$ yields $z^{3}=0$. Then we can assume that $z^{2}=1$, since it can be attained by either replacing operators 2 and 3 or by a change of coordinates in operator 3. Change of the basis makes $x^{2}=y^{2}=0$. Subalgebra condition for the commutator of operators 1 and 3 gives the system of equations

$$
\left\{\begin{aligned}
-\alpha z^{1} & =\gamma y^{1}+\kappa z^{1} \\
-\alpha & =\kappa \\
1 & =\gamma y^{3}
\end{aligned}\right.
$$

that implies $y^{1}=0, y^{3} \neq 0$. Change of the basis makes $y^{3}=1, x^{3}=0$. Subalgebra conditions for the commutator of operators 1 and 4 imply $x^{1}=0$. And, finally, subalgebra condition for the commutator of operators 1 and 2 leads us to the system of equations

$$
\left\{\begin{array}{r}
\mu z^{1}=0 \\
-\alpha=\lambda \\
-1=\mu
\end{array}\right.
$$

that implies $z^{1}=0$. Hence, we obtain subalgebra $\left\{X_{4}+\alpha X_{8}, X_{3}, X_{2}, X_{9}\right\}$.

2) If $y^{9}=z^{9}=w^{9}=0$, the operators of subalgebras are linearly independent if the inequality $y^{1} \neq 0, z^{2} \neq 0, w^{3} \neq 0$ hold true. Then change of the basis leads us to subalgebra $\left\{X_{4}+\alpha X_{8}+\beta X_{9}, X_{1}, X_{2}, X_{3}\right\}$. Thus, there exist two four-dimensional subalgebras in $L_{9}$ with the projection $\left\{X_{4}+\alpha X_{8}\right\}$ on $L_{5}:\left\{X_{4}+\alpha X_{8}, X_{3}, X_{2}, X_{9}\right\}$ is subalgebra 4.2 in Table 3 and subalgebra 4.3 in Table 3: $\left\{X_{4}+\alpha X_{8}+\beta X_{9}, X_{1}, X_{2}, X_{3}\right\}$.

Fifth-dimensional subalgebras in $L_{9}$ with the projection $\left\{X_{4}+\alpha X_{8}\right\}$ on subspace $L_{5}$ have a non-degenerate matrix representation

$$
\left(\begin{array}{cccccc}
1 & \alpha & x^{1} & x^{2} & x^{3} & x^{9} \\
0 & 0 & y^{1} & y^{2} & y^{3} & y^{9} \\
0 & 0 & z^{1} & z^{2} & z^{3} & z^{9} \\
0 & 0 & w^{1} & w^{2} & w^{3} & w^{9} \\
0 & 0 & s^{1} & s^{2} & s^{3} & s^{9}
\end{array}\right)
$$

The operators of subalgebra are linearly independent if the inequalities $y^{1} \neq 0, z^{2} \neq 0$, $w^{3} \neq 0, s^{9} \neq 0$. Then change of the basis leads us to the subalgebra $\left\{X_{1}, X_{2}, X_{3}, X_{9}\right\}$. Hence, there exists one five-dimensional subalgebra in $L_{9}$ with the projection $X_{4}+\alpha X_{8}$ on $L_{5}: X_{4}+\alpha X_{8}, X_{1}, X_{2}, X_{3}, X_{9}$ is subalgebra 5.1 in Table 3.

Now we consider five-dimensional subalgebra 5.1 in Table 2. Six-dimensional subalgebras in $L_{9}$ with the projection $\left\{X_{4}, X_{5}, X_{6}, X_{7}, X_{8}\right\}$ on subspace $L_{5}$ have matrix representation

$$
\left(\begin{array}{ccccccccc}
1 & 0 & 0 & 0 & 0 & x^{1} & x^{2} & x^{3} & x^{9} \\
0 & 1 & 0 & 0 & 0 & y^{1} & y^{2} & y^{3} & y^{9} \\
0 & 0 & 1 & 0 & 0 & z^{1} & z^{2} & z^{3} & z^{9} \\
0 & 0 & 0 & 1 & 0 & w^{1} & w^{2} & w^{3} & w^{9} \\
0 & 0 & 0 & 0 & 1 & s^{1} & s^{2} & s^{3} & s^{9} \\
0 & 0 & 0 & 0 & 0 & t^{1} & t^{2} & t^{3} & t^{9}
\end{array}\right) .
$$


Subalgebra conditions for the commutators of the first three operators give $x^{9}=y^{9}=z^{9}=0$. There are two possibilites.

1) If $t^{9} \neq 0$, transformation $\Gamma$ makes $P_{1}(t)=0$, change of the basis gives $t^{9}=1, w^{9}=s^{9}=0$. Subalgebra condition for the commutator of operators 1 and 6 yields $P_{1}(x)=0$; for operators 2 and 6 it yields $P_{1}(y)=0$; for operators 3 and 6 it does $P_{1}(z)=0$; for operators 4 and 6 it does $P_{1}(w)=0$, for operators 5 and 6 it does $P_{1}(s)=0$. We obtain the subalgebra $\left\{X_{4}, X_{5}, X_{6}, X_{7}, X_{8}, X_{9}\right\}$.

2) If $t^{9}=0$, subalgebra condition for the commutators of operators 1 and 6 leads us to the homogeneous system with non-zero determinant

$$
\left\{\begin{array}{l}
\lambda t^{2}+t^{3}=0 \\
t^{2}-\lambda t^{3}=0
\end{array}\right.
$$

that implies $t^{2}=t^{3}=0$. Change of the basis makes $t^{1}=1$. Subalgebra condition for commutators of operators 2 and 6 gives $\left[X_{5}+y^{1} X_{1}+y^{2} X_{2}+y^{3} X_{3}, X_{1}\right]=-X_{3}=0$, which is impossible. Hence, there are no subalgebras in the second case. Thus, there exists one sixdimensional subalgebra $\left\{X_{4}, X_{5}, X_{6}, X_{7}, X_{8}, X_{9}\right\}$ with the projection $\left\{X_{4}, X_{5}, X_{6}, X_{7}, X_{8}\right\}$ on subspace $L_{5}$ is subalgebra 6.7 in Table 3 .

There exists no seven-dimensional subalgebra in $L_{9}$ with the projection $\left\{X_{4}, X_{5}, X_{6}, X_{7}, X_{8}\right\}$ on subspace $L_{5}$, since subalgebra conditions does not hold. As an example of calculating seven-dimensional subalgebra, we take subalgebra with the projection $\left\{X_{4}, X_{5}, X_{6}, X_{8}\right\}$ which is subalgebra 4.2 in Table 2 . Its matrix representation is

$$
\left(\begin{array}{cccccccc}
1 & 0 & 0 & 0 & x^{1} & x^{2} & x^{3} & x^{9} \\
0 & 1 & 0 & 0 & y^{1} & y^{2} & y^{3} & y^{9} \\
0 & 0 & 1 & 0 & z^{1} & z^{2} & z^{3} & z^{9} \\
0 & 0 & 0 & 1 & w^{1} & w^{2} & w^{3} & w^{9} \\
0 & 0 & 0 & 0 & s^{1} & s^{2} & s^{3} & s^{9} \\
0 & 0 & 0 & 0 & t^{1} & t^{2} & t^{3} & t^{9} \\
0 & 0 & 0 & 0 & u^{1} & u^{2} & u^{3} & u^{9}
\end{array}\right)
$$

Subalgebra conditions for the commutators of the first three operators yield $x^{9}=y^{9}=z^{9}=0$. There exist two options.

1) If $u^{9} \neq 0$, transformation $\Gamma$ gives $P_{1}(u)=0$, change of the basis makes $u^{9}=1, w^{9}=$ $s^{9}=t^{9}=0$. Then we can assume that $t^{2}=1$, since it can be achieved either by replacing operators 5 and 6 or by the change of coordinates in operator 6 . Then change of the basis makes $s^{2}=0$, while subalgebra condition for the commutators of operators 2 and 5 leads us to the homogeneous system with non-zero determinant

$$
\left\{\begin{array}{l}
\gamma s^{1}-s^{3}=0 \\
s^{1}+\gamma s^{3}=0
\end{array}\right.
$$

which implies $s^{1}=s^{3}=0$. That is, the operators are linearly dependent.

2) If $s^{9}=t^{9}=u^{9}=0$, operators $5 \div 7$ of subalgebra are linearly independent if $s^{1} \neq 0, t^{2} \neq 0$, $u^{3} \neq 0$. By change of the basis we obtain the subalgebra $\left\{X_{4}, X_{5}, X_{6}, X_{8}, X_{1}, X_{2}, X_{3}\right\}$. Thus, there exists one seven-dimensional subalgebra $\left\{X_{4}, X_{5}, X_{6}, X_{8}, X_{1}, X_{2}, X_{3}\right\}$ with the projection $\left\{X_{4}, X_{5}, X_{6}, X_{8}\right\}$ on subspace $L_{5}$, which is subalgebra 7.4 in Table 3 .

Let us consider an example of calculating eight-dimensional subalgebra in $L_{9}$ with the projection $\left\{X_{4}, X_{5}, X_{6}, X_{7}, X_{8}\right\}$ on subspace $L_{5}$. The matrix representation of subalgebras reads 
as

$$
\left(\begin{array}{ccccccccc}
1 & 0 & 0 & 0 & 0 & x^{1} & x^{2} & x^{3} & x^{9} \\
0 & 1 & 0 & 0 & 0 & y^{1} & y^{2} & y^{3} & y^{9} \\
0 & 0 & 1 & 0 & 0 & z^{1} & z^{2} & z^{3} & z^{9} \\
0 & 0 & 0 & 1 & 0 & w^{1} & w^{2} & w^{3} & w^{9} \\
0 & 0 & 0 & 0 & 1 & s^{1} & s^{2} & s^{3} & s^{9} \\
0 & 0 & 0 & 0 & 0 & t^{1} & t^{2} & t^{3} & t^{9} \\
0 & 0 & 0 & 0 & 0 & u^{1} & u^{2} & u^{3} & u^{9} \\
0 & 0 & 0 & 0 & 0 & v^{1} & v^{2} & v^{3} & v^{9}
\end{array}\right) .
$$

Subalgebra conditions for the commutators of the first three operators imply $x^{9}=y^{9}=$ $=z^{9}=0$. There are two options.

1) If $v^{9} \neq 0$, transformation $\Gamma$ makes $P_{1}(v)=0$, change of the basis gives $v^{9}=1, w^{9}=s^{9}=$ $t^{9}=u^{9}=0$. As above, we can assume $u^{2}=1$. Then change of the basis implies $t^{2}=0$, and subalgebra condition for the commutator of operators 2 and 6 imply the homogeneous system with non-zero determinant

$$
\left\{\begin{array}{l}
\chi t^{1}-t^{3}=0 \\
t^{1}+\chi t^{3}=0
\end{array}\right.
$$

which yields $t^{1}=t^{3}=0$. That is, the operators are linearly dependent.

2) If $t^{9}=u^{9}=v^{9}=0$, then the last three operators of subalgebra are linearly dependent, if $t^{1} \neq 0, u^{2} \neq 0, v^{3} \neq 0$. Change of the basis makes $P_{1}(t)=\left(\begin{array}{ccc}1 & 0 & 0\end{array}\right) ; P_{1}(u)=\left(\begin{array}{lll}0 & 1 & 0\end{array}\right)$; $P_{1}(v)=\left(\begin{array}{lll}0 & 0 & 1\end{array}\right)$, and also $P_{1}(x)=P_{1}(y)=P_{1}(z)=P_{1}(w)=P_{1}(s)=0$. Subalgebra condition for the commutator of operators 4 and 5 give $\left[X_{7}+w^{9} X_{9}, X_{8}+s^{9} X_{9}\right]=2 X_{7}=\omega\left(X_{7}+w^{9} X_{9}\right)$ that implies $w^{9}=0$. Hence, we obtain 8.3 in Table 3: $\left\{X_{4}, X_{5}, X_{6}, X_{7}, X_{8}+\alpha X_{9}, X_{1}, X_{2}, X_{3}\right\}$.

It is obvious that a nine-dimensional subalgebra with the projection $\left\{X_{4}, X_{5}, X_{6}, X_{7}, X_{8}\right\}$ on subspace $L_{5}$ in $L_{9}$ is algebra $L_{9}$ that can be achieved by change of the basis in the appropriate matrix representation. This is subalgebra 9.1 in Table 3.

By analogy, in accordance with (11) one can calculate subalgebras of all possible dimensions in $L_{9}$ with the projections on subspace $L_{5}$ in Table 2 . For fixed parameters, some of subalgebras are embedded in already found subalgebras in the optimal system, while other subalgebras are independent representatives of OSS. In this way we calculate the optimal system of subalgebras for algebra $L_{9}$. The result is summarized in the following theorem.

Theorem 1. The optimal system of subalgebras for Lie algebra $L_{9}$ with the commutators in Table 1 comprises 117 dissimilar classes, up to internal automorphisms and discrete automorphisms $\varepsilon_{1}$ and $\varepsilon_{2}$, presented in Table 3 where $\alpha, \beta$ are arbitrary constants, $\sigma \neq 0$ is the exponent in the coefficients of equation (1).

Table 3.

\begin{tabular}{|l|l|l|l|}
\hline $\mathrm{r}$ & $\mathrm{i}$ & Basis & Projection on $L_{5}$ \\
\hline \hline 9 & 1 & $1,2,3,4,5,6,7,8,9$ & 5.1 \\
\hline \hline 8 & 1 & $4,5,6,7,1,2,3,9$ & 4.1 \\
\hline & 2 & $4,5,6,8,1,2,3,9$ & 4.2 \\
\hline & 3 & $4,5,6,7,8+\alpha 9,1,2,3$ & 5.1 \\
\hline 7 & 1 & $4,5,6,1,2,3,9$ & 3.1 \\
\hline & 2 & $4,7,8,1,2,3,9$ & 3.2 \\
\hline & 3 & $4,5,6,7,1,2,3$ & 4.1 \\
\hline & 4 & $4,5,6,8+\alpha 9,1,2,3$ & 4.2 \\
\hline \hline 6 & 1 & $4,8,1,2,3,9$ & 2.1 \\
\hline & 2 & $4+\alpha 8,7,1,2,3,9$ & 2.2 \\
\hline & 3 & $7,8,1,2,3,9$ & 2.3 \\
\hline
\end{tabular}




\begin{tabular}{|l|l|l|l|}
\hline $\mathrm{r}$ & $\mathrm{i}$ & Basis & Projection on $L_{5}$ \\
\hline \hline & 4 & $4,5,6,1,2,3$ & 3.1 \\
\hline & 5 & $4+\alpha 9,7,8+\beta 9,1,2,3$ & 3.2 \\
\hline & 6 & $4,7,8,2,3,9$ & 3.2 \\
\hline & 7 & $4,5,6,7,8,9$ & 5.1 \\
\hline \hline 5 & 1 & $4+\alpha 8,1,2,3,9$ & 1.1 \\
\hline & 2 & $4+7,1,2,3,9$ & 1.2 \\
\hline & 3 & $7,1,2,3,9$ & 1.3 \\
\hline & 4 & $8,1,2,3,9$ & 1.4 \\
\hline & 5 & $4,8,2,3,9$ & 2.1 \\
\hline & 6 & $4+\alpha 9,8+\beta 9,1,2,3$ & 2.1 \\
\hline & 7 & $4+\alpha 8,7,2,3,9$ & 2.2 \\
\hline & 8 & $4+\alpha 8+\beta 9,7,1,2,3$ & 2.2 \\
\hline & 9 & $7,8,2,3,9$ & 2.3 \\
\hline & 10 & $7,8+\alpha 9,1,2,3$ & 2.3 \\
\hline & 11 & $4+\alpha 1+\beta 9,7, \sigma 8-9,2,3 ; \beta \neq 0$ & 3.2 \\
\hline & 12 & $4+\alpha 9,7,8+\beta 9,2,3 ; \beta \neq-1 / \sigma$ & 3.2 \\
\hline & 13 & $4+\alpha 1,7, \sigma 8+\beta 1-9,2,3 ; \alpha^{2}+\beta^{2}=1$ & 3.2 \\
\hline & 14 & $4,7,8,1,9$ & 3.2 \\
\hline & 15 & $4,7+1, \sigma 8+\alpha 1+9,2,3$ & 3.2 \\
\hline & 16 & $4,5,6,7,9$ & 4.1 \\
\hline & 17 & $4,5,6,8,9$ & 4.2 \\
\hline & 18 & $4,5,6,7,8+\alpha 9$ & 5.1 \\
\hline \hline 4 & 1 & $1,2,3,9$ & 0 \\
\hline & 2 & $4+\alpha 8,2,3,9$ & 1.1 \\
\hline & 3 & $4+\alpha 8+\beta 9,1,2,3$ & 1.1 \\
\hline & 4 & $4+7,2,3,9$ & 1.2 \\
\hline & 5 & $4+7+\alpha 9,1,2,3$ & 1.2 \\
\hline & 6 & $7,1,2,9$ & 1.3 \\
\hline & 7 & $7+\alpha 9,1,2,3$ & 1.3 \\
\hline & 8 & $8,1,2,9$ & 1.4 \\
\hline & 9 & $8+\alpha 9,1,2,3$ & 1.4 \\
\hline & 10 & $4,8,1,9$ & 2.1 \\
\hline & 11 & $4+\alpha 9,8+\beta 9,2,3$ & 3.1 \\
\hline & 12 & $4+\alpha 1, \sigma 8+\beta 1-9,2,3 ; \quad \alpha^{2}+\beta^{2}=1$ & 2.1 \\
\hline & 13 & $4+\alpha 8,7,1,9$ & 2.2 \\
\hline & 14 & $4+\alpha 9,7+\beta 9,2,3 ; \beta \neq 0$ & 2.2 \\
\hline & 15 & $4+\alpha 1,7+1,2,3$ & 2.2 \\
\hline & 16 & $4+\alpha 8+\beta 9,7,2,3$ & 2.2 \\
\hline & 17 & $4+\alpha(\sigma 8-9)+1,7,2,3$ & 2.2 \\
\hline & 18 & $7,8,1,9$ & 2.3 \\
\hline & 19 & $7,8+\alpha 9,2,3$ & 2.3 \\
\hline & 20 & $7,1+\sigma 8-9,2,3$ & 2.3 \\
\hline & 21 & $7+1, \sigma 8+9,2,3$ & \\
\hline & 22 & $4,5,6,9$ & 3.2 \\
\hline & 23 & $4,7,8,9$ & \\
\hline & 24 & $4+\alpha 9,7,8+\beta 9,1$ & \\
\hline & 25 & $4,5,6,7+\alpha 9$ & \\
\hline & 26 & $4,5,6,8+\alpha 9$ & \\
\hline
\end{tabular}




\begin{tabular}{|l|l|l|l|}
\hline $\mathrm{r}$ & $\mathrm{i}$ & Basis & Projection on $L_{5}$ \\
\hline \hline 3 & 1 & $1,2,3$ & 0 \\
\hline & 2 & $1,2,9$ & 0 \\
\hline & 3 & $4+\alpha 8,1,9$ & 1.1 \\
\hline & 4 & $4+\alpha 8+\beta 9,2,3$ & 1.1 \\
\hline & 5 & $4+1+\alpha(\sigma 8-9), 2,3$ & 1.1 \\
\hline & 6 & $4+7,1,9$ & 1.2 \\
\hline & 7 & $4+7+\beta 9,2,3$ & 1.2 \\
\hline & 8 & $4+7+1,2,3$ & 1.2 \\
\hline & 9 & $7,1,9$ & 1.3 \\
\hline & 10 & $7+\alpha 9,3,2$ & 1.3 \\
\hline & 11 & $7+1,3,2$ & 1.3 \\
\hline & 12 & $8,1,9$ & 1.4 \\
\hline & 13 & $8+\alpha 9,3,2$ & 1.4 \\
\hline & 14 & $8+1,3,2$ & 1.4 \\
\hline & 15 & $4,8,9$ & 2.1 \\
\hline & 16 & $4+\alpha 9,8+\beta 9,1$ & 2.1 \\
\hline & 17 & $4+\alpha 8,7,9$ & 2.2 \\
\hline & 18 & $4+\alpha 8+\beta 9,7,1$ & 2.2 \\
\hline & 19 & $4+\alpha 9,7+\beta 9,1$ & 2.2 \\
\hline & 20 & $7,8,9$ & 2.3 \\
\hline & 21 & $7,8+\alpha 9,1$ & 2.3 \\
\hline & 22 & $7+2, \sigma 8+9,1$ & 2.3 \\
\hline & 23 & $7, \sigma 8+2-9,1$ & 2.3 \\
\hline & 24 & $4,5,6$ & 3.1 \\
\hline & 25 & $4,7+\alpha 1, \sigma 8+9 ; \alpha \neq 0$ & 3.2 \\
\hline & 26 & $4+\alpha 9,7,8+\beta 9$ & 3.2 \\
\hline \hline 2 & 1 & 1,2 & 0 \\
\hline & 2 & 1,9 & 0 \\
\hline & 3 & $4+\alpha 8,9$ & 1.1 \\
\hline & 4 & $4+\alpha 8+\beta 9,1$ & 1.1 \\
\hline & 5 & $4+7,9$ & 1.2 \\
\hline & 6 & $4+7+\alpha 9,1$ & 1.2 \\
\hline & 7 & 7,9 & 1.3 \\
\hline & 8 & $7+\alpha 9,1$ & 1.3 \\
\hline & 9 & $7+2,1$ & 1.3 \\
\hline & 10 & 8,9 & 1.4 \\
\hline & 11 & $8+\alpha 9,1$ & 1.4 \\
\hline & 12 & $8+2,1$ & 1.4 \\
\hline & 13 & $4+\alpha 9,8+\beta 9$ & 2.1 \\
\hline & 14 & $4+\alpha 1, \sigma 8+\beta 1-9 ; \alpha 2+\beta^{2}=1$ & 2.1 \\
\hline & 15 & $4+\alpha 8+\beta 9,7$ & 2.2 \\
\hline & 16 & $4+\alpha 9,7+\beta 9 ; \beta \neq 0$ & 2.2 \\
\hline & 17 & $4+1+\alpha(\sigma 8-9), 7$ & 2.2 \\
\hline & 18 & $4+\alpha(\sigma 8+9), 7+1 ; \alpha \neq 0$ & 2.2 \\
\hline & 19 & $4+\alpha 1,7+1$ & \\
\hline & 20 & $7,8+\alpha 9$ & 2.3 \\
\hline & 21 & $7+1, \sigma 8+9$ & \\
\hline & 22 & $7, \sigma 8+1-9$ & \\
\hline & & \\
\hline
\end{tabular}




\begin{tabular}{|l|l|l|l|}
\hline $\mathrm{r}$ & $\mathrm{i}$ & Basis & Projection on $L_{5}$ \\
\hline \hline 1 & 1 & 1 & 0 \\
\hline & 2 & 9 & 0 \\
\hline & 3 & $4+\alpha 8+\beta 9$ & 1.1 \\
\hline & 4 & $4+1+\alpha(\sigma 8-9)$ & 1.1 \\
\hline & 5 & $4+7+1$ & 1.2 \\
\hline & 6 & $4+7+\alpha 9$ & 1.2 \\
\hline & 7 & $7+1$ & 1.3 \\
\hline & 8 & $7+\alpha 9$ & 1.3 \\
\hline & 9 & $8+\alpha 9$ & 1.4 \\
\hline & 10 & $\sigma 8+1-9$ & 1.4 \\
\hline
\end{tabular}

On figure 1 we show the the distribution histogram for the number $N$ of subalgebras in the optimal system w.r.t. their dimension $r$. One can see that at subalgebras of dimension 3 and 4 we attain the maximum of the number of subalgebras.

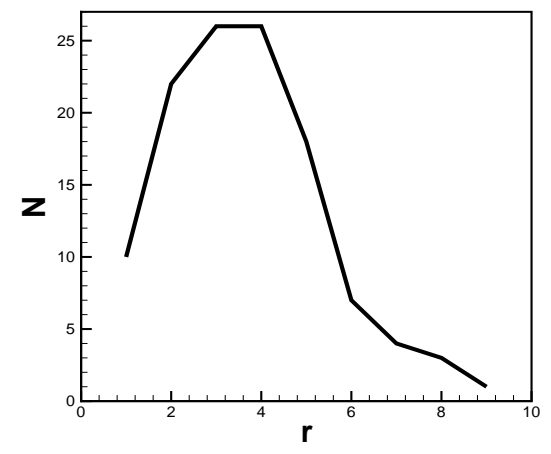

FIGURE 1.

The author expresses his sincere gratitude to Professor Salavat Valeevich Khabirov for the formulation of the problem and permanent attention to this work.

\section{BIBLIOGRAPHY}

1. R.I. Nigmatullin. Fundamentals of Mechanics of Heterogeneous Media. Moscow: Nauka, 1978. 336 pp. (in Russian).

2. V.A. Dorodnitsyn, I.V. Knyazeva, S.R. Svirshchevskij. Group properties of the heat-conduction equation with a source in the two- and three-dimensional cases // Differ. uravn. 1983. V. 19, No. 7. P. 1215-1223. [Diff. Equats. 1983. V. 19. P. 901-908.]

3. L.V. Ovsyannikov. Group analysis of differential equations. Moscow: Nauka, 1978. 399 pp. [Academic Press, New York, 1982.]

4. Yu.A. Chirkunov, S.V. Khabirov. it Elements of symmetry analysis for differential equations of solid state mechanics Novosibirks: Novgorod State Technical University, 2012. 659 pp. (in Russian).

5. S.V. Khabirov. Nonisomorphic Lie algebras admitted by gasdynamic models // Ufimskij matem. zhurn. 2011. V. 3, No. 2. P. 87-90. [Ufa Math. J. 2011. V. 3, No. 2. P. 85-88.]

Aidar Martisovich Ilyasov,

RN-UfaNIPIneft,

Revolutsionnaya str., 96/2,

450078, Ufa, Russia

E-mail: AMIlyasov@gmail.com 